Communication et organisation

$35 \mid 2009$

Repenser la communication dans les organisations publiques

\title{
De l'information utile à la connaissance stratégique : la dimension communicationnelle de l'intelligence économique
}

Nicolas Moinet

\section{OpenEdition}

Journals

Édition électronique

URL : http://journals.openedition.org/communicationorganisation/855

DOI : 10.4000/communicationorganisation.855

ISSN : 1775-3546

Éditeur

Presses universitaires de Bordeaux

Édition imprimée

Date de publication : 1 juin 2009

Pagination : 215-225

ISSN : 1168-5549

Référence électronique

Nicolas Moinet, « De l'information utile à la connaissance stratégique : la dimension communicationnelle de l'intelligence économique », Communication et organisation [En ligne], 35 | 2009, mis en ligne le 01 décembre 2012, consulté le 19 avril 2019. URL : http://journals.openedition.org/ communicationorganisation/855 ; DOI : 10.4000/communicationorganisation.855 
Analyses

\section{Résumé}

Dynamique à la recherche de ses concepts opératoires, l'intelligence économique s'appuie sur des méthodologies issues du terrain qui demandent une assise théorique forte. Pour y être opératoire, le concept d'information doit être compris et utilisé dans un cadre plus systémique qui le relie à l'action via la connaissance. En éveil permanent et tourné vers l'analyse, un dispositif d'intelligence économique mettra alors idéalement en œuvre une communauté stratégique de connaissance dans laquelle la communication apparaît comme centrale.

\section{Mots-clés}

Intelligence économique - information stratégique - connaissance dispositif - communication

\section{Summary}

Constantly on the lookout for new operating concepts, Competitive Intelligence is established on field-based methodologies that require strong theoretical foundations. In order for it to operate, the intelligence concept must be understood and used in a more systemic frame which would link it with action through knowledge. An everworking Competitive Intelligence system, focusing on analysis, will therefore put into motion a strategic community of knowledge wherein communication will appear as the centre of attention.

\section{Key words}

Competitive Intelligence - strategic information - knowledge - system - communication

Nicolas Moinet est Maître de Conférences Habilité à Diriger des Recherches en Sciences de l'Information et de la Communication à l'Université de Poitiers (ICOMTEC). Il est chercheur au CEREGE, dans cette université, responsable de l'équipe de recherche Intelligence Economique et Stratégies de Communication. 


\section{De l'information utile à la connaissance stratégique : la dimension communicationnelle de l'intelligence économique \\ Nicolas Moinet}

nicolas.moinet@univ-poitiers.fr

Définie simplement comme «la maîtrise de l'information stratégique utile aux acteurs économiques» (Juillet, 2004), l'intelligence économique est plus globalement une habileté à comprendre finement et globalement un environnement complexe et à prendre la bonne décision. (D'Almeida, 2001). Maîtrise de l'interaction (Fayard, 2000), elle pose la question de l'action collective ou action organisée dans l'optique du couple information/action dont la clé se trouve dans la génération de connaissances actionnables (Dumas, 1991). Mais pour ce faire, il est nécessaire de passer de l'information à la connaissance en redonnant à la dimension communicationnelle la place qui lui revient. Car s'il n'est certes pas possible d'être intelligent sans information, la réciproque n'est pas automatique : l'information seule ne permet pas d'être intelligent et peut même entraîner une certaine inintelligence (Morel, 2002; Ben Israël, 2004). Ainsi, dès les fondations de l'intelligence économique, tant chez les auteurs anglosaxons que pour les initiateurs du rapport Martre, il va de soi que l'information seule ne permet pas d'agir. Chez Wilensky (1967), l'intelligence est entendue comme le recueil, l'interprétation et la valorisation systématique de l'information pour la poursuite de ses buts stratégiques. Pour l'auteur américain, il ne s'agit pas d'un processus d'accumulation d'informations mais plutôt de production de connaissances, par les gouvernements et les industriels, et quand si nécessaire, dans le cadre de stratégies collectives. Et le véritable levier concurrentiel et stratégique se trouve dans les compétences d'interprétation quand les principaux obstacles sont les rigidités organisationnelles (hiérarchisation et bureaucratisation en particulier). Mais une fois passée cette mise en garde, le quotidien reprend le dessus et c'est le concept d'information qui domine les discours et les pratiques. 
Analyses

\section{Les limites opératoires du concept d'information}

Depuis les travaux de Claude Shannon (1949) et de Robert Wiener (1952), l'idée que l'information est un objet de traitement et un moyen de réduire les incertitudes a la vie dure. L'action est considérée comme une logique permanente d'ajustements qui se réalisent grâce aux rétroactions entre l'émetteur et le récepteur. Cette vision de physiciens et d'informaticiens permettra de nombreux développements dans ces domaines mais posera problème dès lors qu'il s'agit de le transcrire dans les sciences humaines et sociales. Au début néanmoins, ces conceptions trouvent un terrain favorable chez les économistes néoclassiques qui considèrent que l'agent économique est rationnel et que le marché lui fournit naturellement l'information pour l'être (Albertini, Silem, 1983, pp. 80-88). Une idéologie mise à mal par l'école autrichienne et Friedrich Von Hayek pour qui l'information est bien le problème central de l'analyse des processus économiques ${ }^{179}$. Puis Herbert Simon introduit l'idée d'une rationalité limitée : non seulement le décideur dispose d'une information toujours incomplète ne pouvant connaître toutes les conséquences et donc la valeur des différentes possibilités d'action qui s'offrent à lui mais, de plus, sa capacité de traitement de l'information est limitée. L'organisation est alors considérée comme un dispositif permettant de se rapprocher de la rationalité en fournissant aux individus l'information nécessaire et la capacité pour la traiter. Pour James March et Herbert Simon (1958), le processus de décision se compose ainsi de quatre phases : étude de l'environnement, analyse, sélection du mode d'action et décision. L'ensemble fait l'objet d'une évaluation dans une logique d'apprentissage. De plus, March et Simon estiment que l'individu ne peut affronter seul la masse et la complexité des informations et que c'est la synergie des capacités cognitives individuelles qui permet d'y faire face. Une nouvelle conception qui influencera fortement le courant de l'analyse stratégique (Crozier, Friedberg, 1977) pour qui l'organisation - le royaume des relations de pouvoir, d'influence, de marchandage et du calcul - dispose de quatre sources de pouvoir qui correspondent aux différents types de sources d'incertitudes: la maîtrise d'une compétence particulière et de la spécialisation; les relations avec son ou ses environnements; la maîtrise de la communication et des informations; l'existence de

${ }^{179}$ On remarquera que l'article majeur de Von Hayek qui paraît en 1945 dans l'American economic review sous le titre: "The use of knowledge in society» sera traduit dans la Revue française d'économie sous le titre «L'utilisation de l'information dans la société ». 
règles organisationnelles générales; le besoin de réseaux et relais pour l'informer et la représenter (ibid, $p$ 83). La philosophie de l'intelligence économique est là mais il lui reste à percer. Pour cela, elle devra dépasser la notion floue d'information pour introduire celle de connaissance et remettre en question l'illusion qu'il existe un marché de l'information.

L'exemple suivant nous semble bien illustrer l'importance opératoire de cette clarification conceptuelle (Moinet, 2006). Soutenu par les collectivités territoriales, le pôle verrier de Lorraine aide les entreprises et collectivités territoriales à anticiper leurs besoins en innovation et accompagne leur développement en apportant des services adaptés à leurs problématiques, tant en termes d'information stratégique que de communication. La petite équipe est composée d'infomédiaires - qui collectent jusqu'à 2000 informations utiles par an $(50 \%$ sont en anglais, $70 \%$ concernent l'économique, $30 \%$ le technologique) - mais aussi d'experts qui vont les analyser pour permettre aux entreprises de les transformer en connaissances stratégiques. Le pôle propose donc des lettres de veille, organise des événements, valorise les productions locales, notamment sur son site Internet, et met en réseau les acteurs (création de formation dans le domaine du verre par exemple). Puis, fort du succès de ses lettres de veille (trois profils d'abonnement - transformation verre plat, industrie verre plat, industrie verre creux), le pôle verrier propose un abonnement sur-mesure. Plus cher, il propose moins d'informations mais celles-ci sont mieux ciblées. Erreur : le produit ne fonctionne pas alors que les lettres de base continuent à susciter un réel engouement. Une question de prix ? Une enquête montre, en fait, que les lettres de veille sont envoyées à autant de destinataires que le souhaite l'entreprise, et ce sans supplément. Autrement dit, les informations arrivent au même moment à plusieurs services de l'entreprise. Que se passe t-il ensuite? Nul doute que ces informations circulent et font l'objet - consciemment ou non - de discussion et d'échanges croisés. L'information de ces lettres est utile car elle est transformée par l'organisation en connaissance stratégique. Cette hypothèse n'explique-t-elle pas l'échec de l'abonnement sur-mesure? En nécessitant d'affiner ses besoins, ce dernier demandait en effet à l'organisation destinatrice d'externaliser son processus de connaissance, ce qui est tout simplement impossible! Pourtant, rare sont les ouvrages traitant d'intelligence économique - en particulier ceux écrits par des universitaires - qui ne précisent d'emblée les différences qui existent entre donnée, information, connaissance, voire 
Analyses

renseignement. Et qui ignorent superbement le concept clé de communication. Ceci expliquerait-il cela ?

\section{De l'information utile à la connaissance stratégique}

Ainsi que l'avait déjà noté Anne Mayère (1990, p. 285) : "l'information n'existe pas "en soi": c'est un processus engageant activement son "récepteur" qui en est ainsi le co-producteur. L'information acquiert une signification, devient "informationnelle" dans ce processus qui lie étroitement un traitement et son résultat. Et ce traitement engage bien plus que ce que suggère l'analogie informatique (...) En cela, le traitement de l'information est aussi celui d'une "information sur l'information" qui lui est associée et qui concerne la décidabilité de l'information. ». C'est alors qu'apparaît la notion de connaissance comme produit individuel et collectif issus d'informations et guidé par une vision commune du but à atteindre et du sens à donner à l'action. L'économiste Guy Massé propose ainsi de considérer que «la gestion de l'information est avant tout un problème culturel de légitimation d'une envie ou d'un besoin, de compréhension qu'elle est moins un coût qu'une valeur, que la rétention doit céder le pas au partage pour aboutir à l'intelligence. Celle du groupe : la "co-naissance »; celle du projet, de la méthode, des outils : la " connaît-sens»» (2001, p. 201). Et de conclure : «La connaissance est finalisée dans l'action, elle traduit le passage d'un savoir à un savoir-utiliser. La connaissance devient renseignement par rapport à une finalité, elle enseigne «sur» un objectif, elle enseigne «pour» une action.» (Ibid., p 179). D'où l'idée que l'intelligence économique ressemble à un système sensoriel de perception vigilante, de compréhension cohérente, de persuasion permanente et d'action pertinente.

Reste que les notions d'information et de connaissance restent souvent assimilées. S'appuyant en particulier sur les travaux de Dominique Foray (2000), Jean-Louis Levet (2001) les différencie ainsi : «la connaissance est d'abord une capacité d'apprentissage et une capacité cognitive, alors que l'information reste un ensemble de données formatées et structurées. La propriété essentielle de la connaissance est de pouvoir par elle-même engendrer de nouvelles connaissances, alors que la reproduction de l'information s'effectue simplement par duplication. La connaissance est composée non seulement d'informations à caractère public, mais aussi de savoirfaire inexprimables formellement et donc difficilement transférables. Ils sont incorporés dans les individus et les organisations, autrement dit, ils ne peuvent pas être isolés de leur environnement. La création 
de connaissances nouvelles apparaît, par conséquent, comme un processus d'apprentissage ». (Levet, 2001, pp. 40-41). En accord avec la vision systémique et remarquant que la plupart des auteurs en intelligence économique définissent des objets plutôt que des processus, nous reprenons le schéma développé par le consultant Pierre Chapignac (1994) à l'époque du rapport Martre.

\section{PENSER L'ACTION}

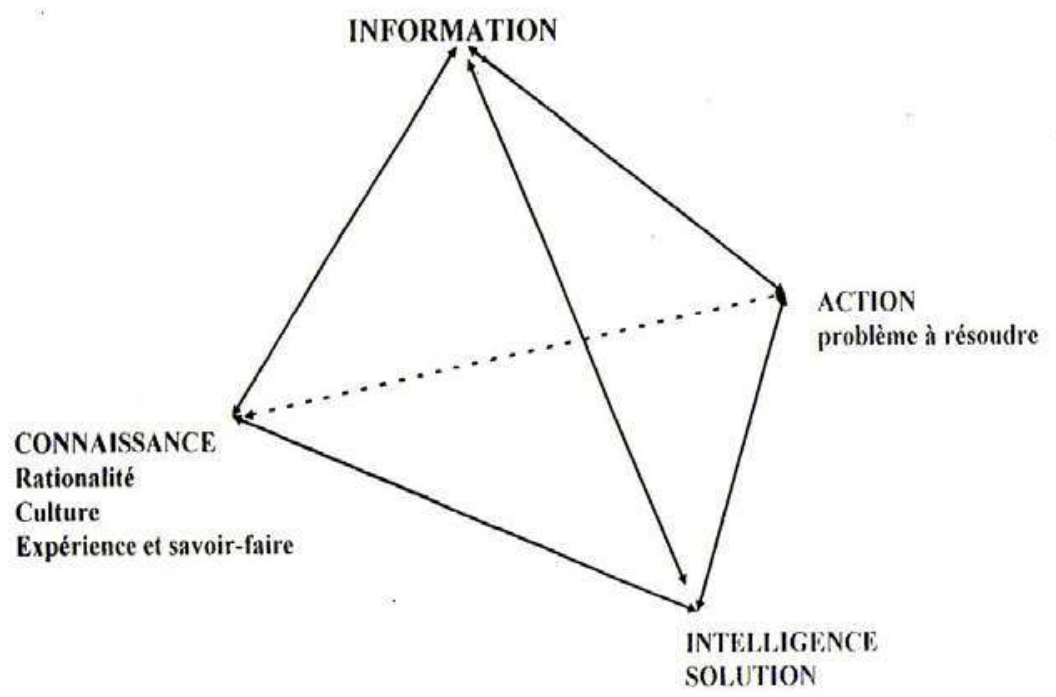

Travaillant sur la question cruciale de la valeur de l'information, Pierre Chapignac estime que celle-ci est déterminée par son intégration dans un ensemble que l'on peut nommer «l'action pensée ». En d'autres termes, la valeur de l'information se définit dans le contexte de l'action. Si cette valeur peut se juger bien évidemment sous l'angle du contenu, elle peut l'être également sous l'angle de la redondance, de la diversité et de la quantité. La valeur de l'information est déterminée par son usage et non par sa nature ainsi que par le service rendu. Puis l'auteur établit un lien fonctionnel entre information et connaissance: "La valeur de l'information est proportionnelle à la connaissance intégrée. Cette connaissance est d'autant plus apporteuse de valeur qu'elle est diversifiée" ". (Chapignac, 1994). La valeur de l'information est ainsi déterminée par le niveau de partage ainsi que par la quantité et la qualité des échanges. Du schéma, retenons l'idée qu'aucun des éléments ne peut être simplement relié à un autre sans que l'on considère l'ensemble. S'il existe des situations où les liens sont directs et courts (le problème trouve par exemple sa solution sans qu'il soit nécessaire d'intégrer de 
Analyses

nouvelles informations via le système de connaissance), celle qui nous intéresse - la décision stratégique déterminante porteuse d'un avantage relatif - fait en général appel au système. La stratégie peut alors être définie comme "l'art d'utiliser les informations qui surviennent dans l'action, de les intégrer, de formuler soudain des schémas d'action et d'être apte à rassembler le maximum de certitudes pour affronter l'incertain» (Morin, 1990, p 178). L'intuition si chère au chef d'entreprise n'est sans doute pas autre chose que ce système masqué (consciemment ou non) et le «chainon manquant» de l'intelligence économique entre information et connaissance pourrait bien être le processus de communication. «Dans l'organisation, la communication est l'acte qui met en relation les composants de l'organisme. Il n'y a pas de communication sans organisation; pas d'organisation sans communication et pas de communication sans information $\gg$. (Dumas, 1991, p 36).

Après une thèse de Doctorat en sciences économiques, Antoine Saïd, ingénieur en électronique et en télécommunications, se voit confier la mise en place d'un système de veille technologique et stratégique chez SFR. Rapidement, il se rend compte que la principale difficulté est humaine : comment amener les acteurs du système d'information à mobiliser et diffuser la connaissance. Un aspect souvent négligé par les théoriciens de l'intelligence économique, notamment en France. Dans une optique individualiste, les recettes individuelles l'emportent sur le management collectif. Pour Antoine Saïd (2004), il manque aujourd'hui encore un chaînon entre la conception et l'application des méthodologies issues de l'intelligence économique et la réalité de la performance de l'entreprise. S'inspirant de la littérature anglo-saxonne et d'expériences de terrain, il estime que l'intelligence économique est surtout un processus contribuant, en construisant du sens, à modifier les représentations que les acteurs de l'organisation ont de leur environnement. Cette dimension collective réside dans le triptyque : appropriation - interprétation - action. En ce qui concerne l'acquisition, l'assimilation et la transformation de l'information, Antoine Saïd souligne l'importance de la notion de capacité d'absorption des personnes incluses dans le système d'intelligence économique. Cette capacité consiste à reconnaître la valeur d'une nouvelle information, à l'assimiler et à l'exploiter (connaissance) à des fins stratégiques. En somme, il s'agit de passer d'une conception de l'information utile (Martre, 1994) au pouvoir de capacité d'absorption pour produire de la connaissance stratégique. 
De l'information utile à la connaissance stratégique...

\section{La communication, dimension oubliée de l'intelligence économique}

«Pour ma part, explique Edgar Morin, je n'ai jamais considéré l'information seule, mais toujours encadrée par l'organisation et par la communication. » (Laulan, Perriault, 2007, p. 187). L'information ne devient stratégique que si elle apporte de l'eau au moulin de la connaissance. Toute la difficulté est alors de créer un cycle vertueux d'apprentissage organisationnel. La clé est donc politique. Car qu'estce que cette intelligence dont nous parlons tant si ce n'est justement la capacité à relier ce qui est épars et transformer l'information en connaissance, à piloter une dynamique d'apprentissage (Senge, 1991) en étroite relation avec son environnement mais aussi à désapprendre ce qui pourrait paralyser la pensée et l'action (Baumard, 1995).

L'intelligence étant une affaire collective, c'est bien d'une question de culture qu'il s'agit. C'est pourquoi les outils phare de l'intelligence économique que sont les rapports d'étonnement ou les missions de benchmarking semblent d'une simplicité parfois déconcertante. Mais dès lors qu'il s'agit de les mettre en œuvre, ils impliquent un management participatif et un travail en réseau qui soient guidés par une réelle volonté de donner du sens. Il s'agit là d'une conception constructiviste et interactionniste de l'organisation telle qu'a pu la développer Karl Weick. En rupture avec une vision rationnelle de l'organisation qui fait reposer celle-ci sur des caractéristiques objectives (taille, technologie, environnement, etc.), ce professeur américain de psychologie et de science des organisations considère que l'organisation est en mouvement et qu'elle ne cesse de se construire et de se déconstruire en raison des multiples interactions qui œuvrent en son sein: communication, interprétation, adaptation mutuelle. Dans son ouvrage de référence The social psychology of organizing $(1969,1979)$, Karl E. Weick explique ainsi que les organisations ne peuvent réagir qu'à des éléments d'environnement institués par les acteurs à travers leur activité cognitive. L'élaboration de sens est collective et résulte de cycles d'enactment ou énaction (Varela, 1988): un acteur $\alpha$ (individu ou groupe) propose une interprétation qui est acceptée, rejetée ou modifiée par $\beta$ (interaction) et amène $\alpha$ à abandonner, réviser ou maintenir son choix (doubleinteraction). Ces cycles de comportement interreliés permettent d'aboutir à une construction collective de sens et de faire face à l'imprévu. Ainsi, la probabilité de survie d'une organisation augmente lorsque les variations et les possibilités se multiplient et que les essais et erreurs deviennent plus divers et moins typiques, que les individus se répètent moins et que la créativité est encouragée. Pour Weick 
Analyses

(1995), ce processus de construction de sens est déclenché par l'inattendu, orienté vers l'action et sensible au contexte. Une bonne illustration en est donnée par le redressement des chantiers navals de l'Atlantique (Moinet, 2006).

En 1997, les chantiers de l'Atlantique doivent faire face à une souscharge de travail due à l'incapacité de prendre des commandes sans subvention étatique. Par chance, le nouveau directeur qui ne connaît rien au secteur commence par une analyse stratégique. La segmentation du marché apporte une première bonne nouvelle : il existe des gros marchés où les Asiatiques sont peu présents et où Alstom Marine est bien positionné. Puis, il se livre à la décomposition complexe du coût d'un navire (par exemple, il y a pas moins de 21 variables dans le coût d'une coque). Sur les 160 variables, 80 sont sélectionnées qui vont faire l'objet d'études de benchmarking avec les concurrents, les fournisseurs, les brokers, les clients, les experts internes, etc. Au total, 200 interviews sont réalisées avec une question clé : comment chiffrez-vous ces coûts ? Et l'analyse comparée est sans appel : Alstom Marine est en moyenne 20 à $25 \%$ plus chère que ses concurrents. Une analyse des forces et faiblesses est menée parallèlement. Principales faiblesses : le manque de compétitivité et la dépendance aux subventions. La réponse est alors donnée par la mise en œuvre du plan CAP 21 dont les trois objectifs sont : améliorer la performance (30\% de réduction de coût dans les trois ans), devenir leader mondial des paquebots et augmenter le volume de production. Les chantiers de l'Atlantique sont sauvés. Mais l'intelligence économique, c'est aussi l'évolution de la culture de l'entreprise. Point positif chez Alstom Marine: l'attachement du personnel pour un produit fascinant. Point négatif «très français » selon Patrick Boissier : une certaine arrogance qui fait que l'on sait mieux que le client ce qui est bon pour lui. Un exemple : un client voulait une drague avec une certaine ouverture. L'équipe d'ingénieurs a réussi à démontrer «par $\mathrm{A}+\mathrm{B}$ » et la réalisation d'un mémoire d'une centaine de pages que ce n'était pas possible. Oui mais voilà explique l'ancien Directeur Général des chantiers navals de l'Atlantique : "un de nos concurrents ne savait pas que c'était impossible et il l'a fait ». Pour modifier cet état d'esprit, Alstom va avoir une chance extraordinaire : le besoin d'embaucher au plus vite 600 personnes. Vu le nombre, ces nouveaux embauchés viennent de tous les horizons et apportent avec eux l'excellence de domaines a priori éloignés, mais a priori seulement. L'innovation n'a pas de limites. Exemple: les derniers paquebots sont maintenant équipés de piscines à vagues pour faire... du surf! En interne, note Patrick Boissier, l'important est d'obtenir 
De l'information utile à la connaissance stratégique...

l'adhésion du personnel: «Il faut communiquer et entretenir la flamme».

Evoquée bien sûr, effleurée sans doute mais rarement intégrée aux réflexions et pratiques de l'intelligence économique, la communication est le parent pauvre des pratiques d'intelligence économique et l'analyse communicationnelle apparait comme la grande absente des recherches sur la question Pourtant, ré-introduire l'analyse des processus communicationnels dans l'étude des démarches d'intelligence économique nous entraîne nécessairement à distinguer l'information et la connaissance. Une distinction aussi essentielle qu'oubliée.

Mais est-ce totalement un hasard? L'écart entre la qualité de l'information ou les biais cognitifs et le discours velléitaire sur les décisions qui s'imposent est, en général, important. Car les processus identifiés relativisent la toute-puissance des décideurs et modifient la «géographie » décisionnelle: qui décide vraiment? Où est le pouvoir? Comment se fabrique la stratégie? «Pour l'observateur extérieur, l'écart est frappant et on peut être tenté d'y voir une touchante illusion. Mais sans doute une organisation où les managers auraient conscience de ne pas peser significativement sur le cours de l'action et abandonneraient tout effort pour décider serait-elle promise à une rapide décadence. Il faut admettre que les décideurs ne décident pas mais que leur travail de décideur a cependant un effet réel dans la fabrication des décisions (de l'action)». (Bellier, Laroche, 2005, p 19).

\section{Bibliographie}

Albertini J-M., Silem A., Comprendre les théories économiques, Paris : Seuil, 1983.

BAUMARD P., «Des organisations apprenantes? Les dangers de la consensualité », in Revue Française de Gestion, $\mathrm{n}^{\circ}$ spécial Les chemins du savoir de l'entreprise, septembre-octobre, 1995, pp. 49-57.

Bellier S., Laroche H., Moi, Manager, Paris : Dunod, 2005.

BEN-ISRAEL I., Philosophie du renseignement : logique et morale de l'espionnage, Paris : Editions de l'éclat, 1999.

Crozier M., Friedberg E., L'acteur et le système, Paris : Seuil, coll. Points, 1997.

D’Almeida N., Les promesses de la communication, Paris : Presses Universitaires de France, 2001. 
Analyses

DUMAS P., Information et action, Habilitation à Diriger des Recherches, Université du Sud Toulon-Var, 1991.

FAYARD P., La maîtrise de l'interaction (l'information et la communication dans la stratégie), Ed. 00h00.com., 2000.

JUILlET A., « $\mathrm{Du}$ renseignement à l'intelligence économique », in Défense Nationale, ${ }^{\circ} 12,2004, \mathrm{pp}$ 7-20.

Laulan A-M., Perriault J., "Interview d'Edgard Morin », in Hermès 48 : Racines oubliées des sciences de la communication, Paris : Editions du CNRS, 2007, p 185-187.

LEVET J-L., L'intelligence économique: mode de pensée, mode d'action, Paris : Economica, 2001.

MARCH J., SimON H., Organizations, New Yok : John Wiley \& sons, 1958.

MARTRE H., Intelligence économique et stratégie des entreprises, Rapport du groupe de travail du Commissariat Général du Plan, Paris : La Documentation Française, 1994.

MASSE G., THIBAULT F., Intelligence économique : un guide pour une économie de l'intelligence, Bruxelles : De Boeck, 2001.

MAYere A., Pour une économie de l'information, Lyon : Editions du CNRS, 1990.

MOINET N., «PME et intelligence stratégique », in Veille, décembre 2006 - janvier 2007, pp. 19-27.

MOREL C., Les décisions absurdes (Sociologie des erreurs radicales et persistantes), Paris: Gallimard, Bibliothèque des sciences humaines, 2002.

SAÏD A., Rôle de l'intelligence économique dans la stratégie de l'entreprise, Thèse de Doctorat en sciences de gestion, 2004.

SENGE P., La Cinquième discipline, Paris : Editions First, 1991.

VARELA F.J., Autonomie et connaissance, essai sur le vivant, Paris : Seuil, 1998.

WEICK K.E., The social psychology of organizing, New-York: Random House, 1979.

WeICK K.E. (1995), Sensemaking in Organizations, California : Sage Publications. 
De l'information utile à la connaissance stratégique...

WIENER R., Cybernétique et société, Paris : Editions des Deux Rives, 1952.

WILENSKY H., Organizational Intelligence: Knowledge and Policy in Government and Industry, New York: Basic Books, 1967.

Wolton D., « De l'information aux sciences de la communication », In Hermès 48 : Racines oubliées des sciences de la communication, Paris : Editions du CNRS, 2007, p 189-202. 